\title{
Student as an investigator: simulation on optic synergetics
}

Boris Poizner, Alexey Arshinov, Renat Mudarisov, Natalyia Starova

Boris N. Poizner, Alexey I. Arshinov, Renat R. Mudarisov, Natalyia A. Starova, "Student as an investigator: simulation on optic synergetics," Proc. SPIE 2525, 1995 International Conference on Education in Optics, (13 October 1995); doi: 10.1117/12.224076

SPIE Event: SPIE's 1995 International Symposium on Optical Science, Engineering, and Instrumentation, 1995, San Diego, CA, United States 
Student as an investigator: simulation on optic synergetics

Boris Poizner, Alexey Arshinov, Renat Mudarisov, Nataliya Starova

Tomsk University, Department of Radiophysics

Tomsk, Russia, 634050

\begin{abstract}
$\underline{\text { ABSTRACT }}$
The authors take over the following tasks: development of fundamental creative styles of student's thinking; study of some morphogenesis phenomena; the mastery of computer technology of investigations on optical synergetics by the students.

The authors have elaborated the system of training-investigating problems presented to students. Each student is put into problem situation of an investigator, solving a new problem concerning the optical morphogenesis in a ring four-mirror interferometer, containing nonlinear medium and linear two - dimensional large - scale transformator of the light field (in the feedback circuit). The phase shift distribution in the cross section of the laser beam is described by nonlinear parabolic equation with displaced space argument. The student must study processes of the optical structures generation by means of the simulation. The content of the training - investigating problems foresees various tasks for the student, for instance an explanation of the simulation results, predictions about the structures shape and behavior, test of the student hypothesis, modifcation of the physical model.
\end{abstract}

$\underline{\text { Keywords: }}$ creative styles, problem situation, simulation, optical structures, optical morphogenesis, synergetics.

\title{
1. EDUCATIONAL AIMS AND METHODS
}

In authors opinion, the university education must develop the features of creative thinking in a student and form holistic - evolutional world outlook.

It is well known that fundamental creative styles of a thinking of a investigator are following: model - symbol, nonlinear (or so-called synergetical), system style. A problem situation promotes to development of the creative thinking and the creative activity - this thesis served as the basis of the pedagogical strategy.

In order to form holistic - evolution vision the future opticians are charged with the study of some synergetical phenomena in nonlinear system by means of a computer simulation. Thus the mastery by the students of this modern technology of science investigations in the sphere of optical synergetics is realized. So, there are three general educational aims: the progress of the student creative ability, becoming of his holistic - evolutional consciousness, a growth of his competence in the sphere of the simulation.

\subsection{Variant of individual working}

In order to reach the mentioned tasks the authors have elaborated the system of training - investigating problems (TIP) presented to students. The content of the each TIP envisages the following work performing by student: composition or modification or interpretation of a physical model; constructing of differential equations as a mathematical model; composition of a computer program; computer simulation; discussion of simulation results and conclusions in a form of "science propositions", which the student must advocate.

By this means each student is put into position of an investigator, solving a new problem, which decision is absent in a literature.

\subsection{Variant of group working}

If the teacher has good reason to develop the student culture of scientific communication it is convenient to organize the "research group". The student - "theorist", the student - "programist" and student - "chief" may be this group. The teacher mission is to present a "technical assignment" for the "research group". The function of "chief" is to formulate the corresponding investigation matter; "theorist" must set up a mathematical model of an optical selforganization processes; the 
task of the "programist" is to realize the computer simulation. The "chief" must control over the work of the group and prepare the "report on the investigations". He also advocates the report in a role situation when the teacher acts as a "customer".

\section{LASERS AND OPTICAL SYNERGETICS}

It is common knowledge that synergetics origin is closely allied to the studies of the processes in a laser. G.Haken, one of the founders of synergetics, considers a laser as a system prototype, which during the process of selforganization creates complicated space - time structure. The transition from non - coherent (spontaneous) radiation to coherent laser radiation provides one of examples of selforganization.

\subsection{Light forms optical structures}

Laser as an object of investigation synergetics gives place to laser as factor gave birth to new classes of selforganization processes, in the present state of the synergetics.

Among the new classes of processes are shaping phenomena in the ring optical rescnator (interferometer) containing one or several space separated nonlinear media. The ring nonlinear interferometers were suggested and investigated by S.Akhmanov, M.Vorontsov a.o. [1] (Moscow State University) experimentally.

Studies of the generation of structures (distribution of the phase shift or the intensity at the cross - section of laser beam) form the individual branch of synergetics called optical synergetics. It turns out very convenient "proving ground" for experimental observation of the optical structures morphogenesis and dynamics.

\subsection{Simplified scheme of nonlinear ring resonator}

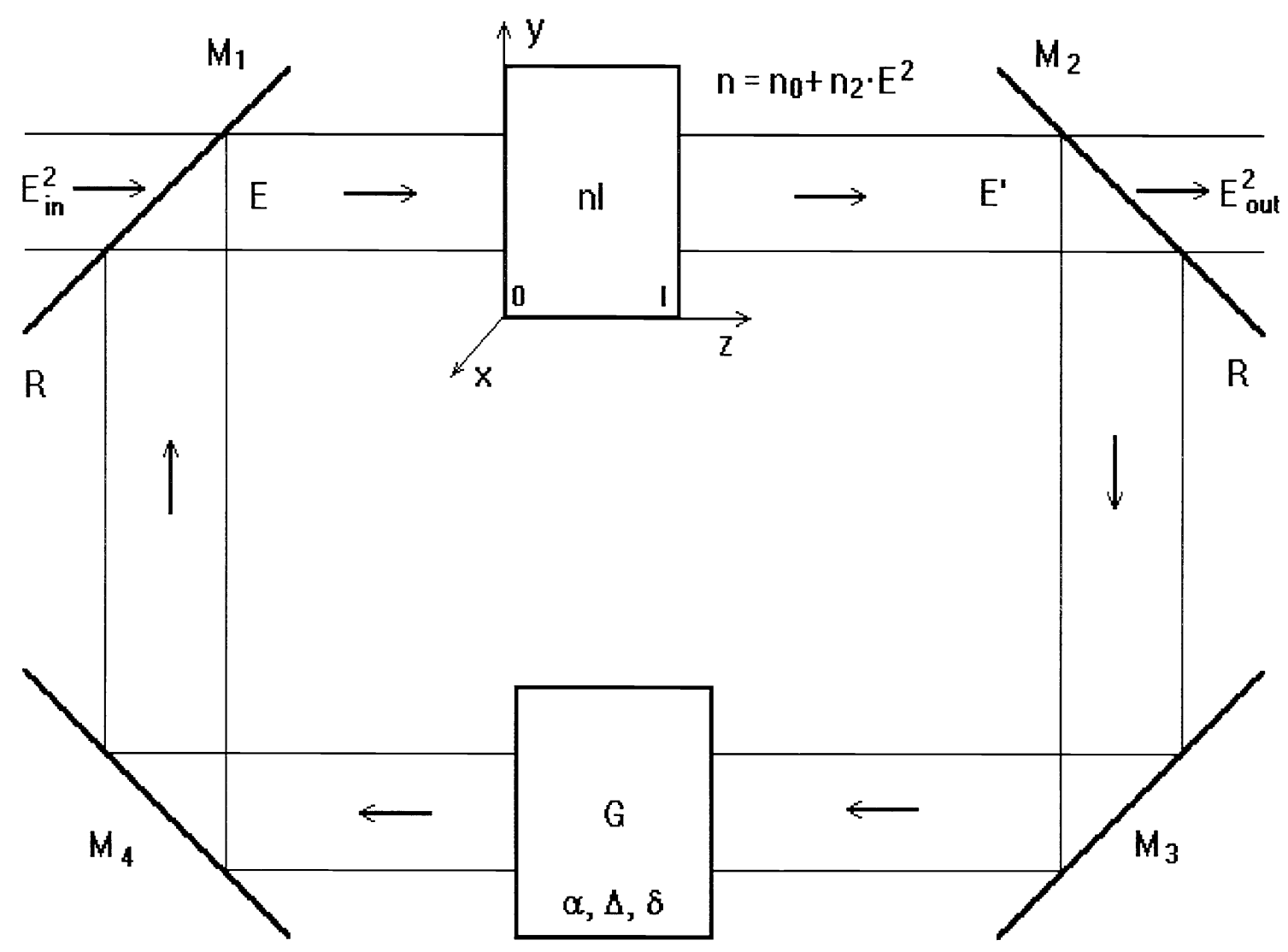

Fig. 1. Scheme of the ring interferometer [1]. 
The Fizeau interferometer containing a liquid crystal whose refractive index depends on a light power (Kerr nonlinear medium) and realizing linear two - dimensional large - scale transformation of light field in the feedback circuit (Fig. 6.19 in book [1]) is very convenient for computer simulation of the evolution of phase shift distribution at the cross section laser beam [2-4]. Condition and pecular features of the stationar regimes of optical structures generation have been of our main interest here.

The main components of the ring interferometer simplified scheme are presented in Fig. 1, where the following designations are used:

- $E_{\text {in }}$ - the complex amplitude of the light field of input laser beam;

- $\mathrm{M}_{1,2,3,4}$ - the mirrors of the interferometer;

- $\mathrm{nl}$ - the layer of the nonlinear medium having thickness $\mathrm{l}$;

- $\mathrm{G}$ - the optical systems provided linear transformation of the field due to which the path of the light ray starting at a point $(\mathrm{x}, \mathrm{y})$ arrives to a point $\left(\mathrm{x}^{\prime}, \mathrm{y}^{\prime}\right)$ upon the total passage of the interferometer;

- $\mathrm{E}_{\text {out }}$ - the complex amplitude of the light field of output laser beam.

The shaping of the two - dimensional patterns in the nonlinear interferometer by student performing the TIP. As a rule this is university student - radiophysicist or physicist of 8-th or 9-th semester.

\section{MODEL OF OPTICAL STRUCTURES EVOLUTION}

Evolution of the phase shift $\mathrm{u}(\mathrm{x}, \mathrm{y}, \mathrm{t})$ distribution in the plane $(\mathrm{x}, \mathrm{y})$ of cross section of laser beam in the Fizeau interferometer is described [1] by nonlinear parabolic equation with displaced space argument:

$$
\cdot \tau \cdot \frac{\partial u(x, y, t)}{\partial}=d \cdot \Delta_{\perp} u(x, y, t)-u(x, y, t)+K \cdot\left\{1+\gamma \cdot \cos \left(u\left(x^{\prime}, y^{\prime}, t\right)+\varphi_{0}\right)\right\} \cdot
$$

where $\tau$ - relaxation time of the nonlinear part of the liquid crystal refractive index $n(x, y, t)=n_{0}+n_{2} E^{2}(x, y, t) ; \Delta-L a p l a s i a n ; d$ - diffusion coefficient; $K=k \cdot l \cdot(l-R) \cdot n_{2} \cdot E_{\text {in }}^{2} ; \varphi_{0}=k \cdot l \cdot n_{0} ; \mathrm{k}$ - wave number; $R$ - reflectivity of mirrors $\mathrm{M}_{1}, \mathrm{M}_{2}$ of Fizeau interferometer (Fig. 1); the coordinates are normalized by the radius of the laser beam.

The displaced coordinates $\left(x^{\prime}, y^{\prime}\right)$ of the light field, undergoing to the linear space transformation in the feedback circuit, and coordinates $(\mathrm{x}, \mathrm{y})$ are related by the following expressions:

$$
\begin{aligned}
& x^{\prime}=(x \cdot \cos (\Delta)+y \cdot \sin (\Delta)) / \alpha+\delta \\
& y^{\prime}=(y \cdot \cos (\Delta)-x \cdot \sin (\Delta)) / \alpha,
\end{aligned},
$$

where $\alpha$ - scale changing of the laser beam, $\Delta$ - light field turn in feedback circuit, $\delta$ - field shift in plane (x,y). By this means it takes place the interaction of the optical fields located at different points of the cross section of the laser beam. So the following phenomena take place in the interferometer:

- the electrooptical (Kerr) effect in the liquid crystal, described by the nonlinearity coefficient $\mathrm{K}$ in the equation (1);

- the interference of the input beam field and field undergoing to the space transformations (the stretching or compression $\alpha$ of the beam, the turn $\Delta$ about the beam axis etc. ), described by the visibility $\gamma$ in (1), where interference field determines the electroopical effect;

- the diffusion of the liquid crystal molecules polarized by the light field measured by the coefficient $d$ in (1);

- the relaxation of the nonlinear part of the liquid crystal refractive index $n(x, y, t)$.

The equation (1) describes the evolution of the full phase shift distribution $u(x, y, t)=k \cdot 1 \cdot n(x, y, t)$ of the light wave in the medium of length 1 , whose nonlinear part is in proportion to the light power. Model (1) suggests that the plane simple harmonic wave makes only one passage across the Fizeau interferometer: $\gamma^{2}<<1$; the delay of the light field undergoing the linear space transformations is absent; the light propagation is treated as a geometrical optics approximation.

According to the evident physical sense the boundary conditons have form

$$
\frac{\partial u(x, y, t)}{\partial x}=\frac{\partial u(x, y, t)}{\partial y}=0
$$


The initial conditions may be various (the twelve variants may be suggested), for instance, it may represent the "chess desk":

$$
u(x, y, 0)=\sin (4 \cdot \pi \cdot x) \cdot \sin (4 \cdot \pi \cdot y)+1 .
$$

\section{SIMULATION OF THE OPTICAL SYNERGETICS PROCESSES}

The simulation stages performed by the student differ by a complication level.

\subsection{The content of the TIP}

The content of the TIP foresees the following tasks for a student:

- to explain the physical phenomena and processes (its nature and role) take into account ir deducing of the equation (1);

- to make an estimation of the applicability of the physical model (1);

- to modify the physical model (1) in connection with the conditions stated by the teacher (for example, the interferometer contains two different nonlinear media);

- to construct the differential equation corresponding to the modified model;

- to compose the computer program or its fragment connected with the modification of the equation;

- to propose the alternative physical model of the phase shift dynamics based on the alternative optical scheme;

- to advocate this variant conclusively.

A numerical decision of the equation (1) is independent and complicated (for the student) task. The authors use here: absolutely steady implicit scheme with a constant step in time (t); Laplacian and boundary conditions approximation on an uniform net having a second order of an accuracy; the decision of difference equations (arising on the every step in time) by sweep method. As the authors experience shows it is appopriately in order to student was introduced to choice principles of numerical decision methods of (1) and corresponding guidebooks.

\subsection{Verification of the model (1)}

According to a sense of the computer simulation a mathematical model must by verified by means of a comparison with laboratory experiments results. Because the first step of the simulation performed by the student is so called calibration procedure of the model (1). The student must be acquanited with the optical pattern generation in the nonlinear ring interferometer which has been observed by S.Akhmanov, M. Vorontsov with colleagues [1] during laboratory experiments. Later the student must by convinced that the regularities derived from the simulation is confirmed by the experemental observations of the authors [1]. For example the student can verify that, when the light field (in the feedback circuit) is turned about a wave vector through angle $\Delta$ under the invariable laser beam size in the feedback circuit $(\alpha=1)$ and in the absence of the interferometer mirror shift $(\delta=0)$, the optical pattern is "multipetal" structure. Id est the structure looks as a camomile (Fig. 2 - 4). If the angle $\Delta$ satisfies the condition:

$$
n=m \cdot 180^{\circ} / \Delta
$$

where $\mathrm{n}$ and $\mathrm{m}$ are integers, then the multipetal structure stands motionlessly and a number of the its petals is equal $\mathrm{n}$.

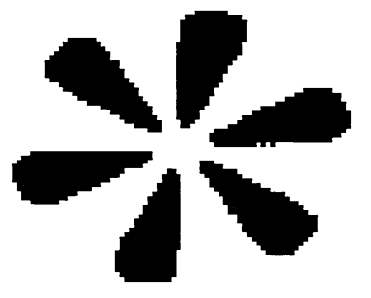

Fig. 2.

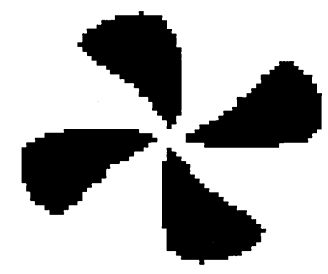

Fig. 3.

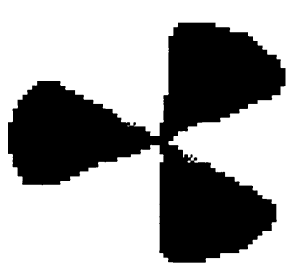

Fig. 4. 
The structure in the Fig. 2 . forms when $\Delta=30^{\circ}, \mathrm{d}=0.005, \delta=0, \alpha=1, \mathrm{~K}=4.08$, th e initial conditions correspond to the case (2). If $\Delta=45^{\circ}$ under the mentioned parameters the structure has the shape shown in Fig. 3. The structure in Fig. 4 generates when $\Delta=60^{\circ}$ and $\mathrm{K}=2.36$.

But if the angle $\Delta$ not satisfies the condition (3) then the multipetal structure rotates in the plane (x,y) of the laser beam cross section continuously. The rotation frequency of the structure depends on the nonlinear interferometer parameters. The change of the angle $\Delta$ in the range including the value of $\Delta$ satisfying (3) (for example from $40^{\circ}$ to $50^{\circ}$ ) causes the reverse of the multipetal structure rotation direction.

The student can choose another type of the structure in order to make sure that model (1) describes a general regularity of the optical patterns constructions and dynamics correctly. Here a situation is possible, when the results of the simulation and the experimental data don't coincide completely. In that case the student must propose the model modification and test the efficiency of this improvement or he must justify an impossibility take into account some physical factors in limits of approximations made when the model (1) was built.

Thus the student can realize capabilities of the morphogenesis model (1) and its truth degree.

\subsection{Student predictions about the structure shape and test of hypothesis}

Main part of the TIP is the simulation undertaken by the student for the purpose of the test of hypothesis promoted by him. According to an idea of the TIP the student must predict the dependence of the structure configuration as a whole or/and the important details of its shape on the initial distribution of light field phase shift $u(x, y, 0)$ and on the parameters of the nonlinear interferometer. Then the student must test hypothesis by means of the simulation and interpretate the results.

For example, the student must predict how the morphogenesis rate of the structure type "optical bistability" depends on the nonlinearity parameter $\mathrm{K}$ and the visibility $\gamma$.

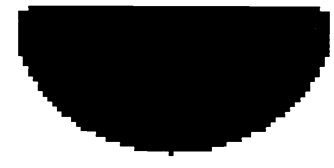

Fig. 5 .

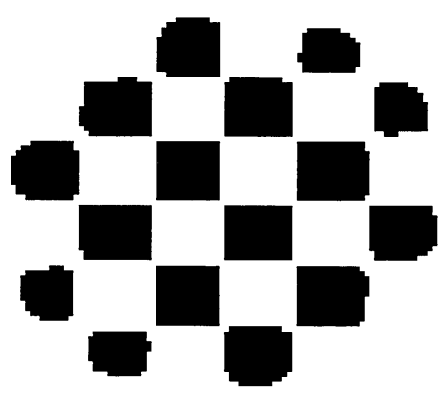

Fig. 6.

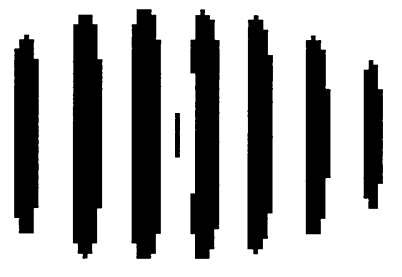

Fig. 7.

The structure type "optical bistability" shown in Fig. 5 forms when $\Delta=180^{\circ}, \mathrm{d}=0.001, \delta=0, \alpha=1, \mathrm{~K}=2.36$. If $\Delta=$ $90^{\circ}, \mathrm{d}=0.001, \delta=0, \alpha=1, \mathrm{~K}=2.36$ and relation (2) performs the structure type "optical multistability" generates (Fig.6). The structure type "shift nonstability" (Fig. 7) forms when $\Delta=0, d=0.001, \delta=0.1, \alpha=1, K=2.36$.

Another example: the student must predict the features of the structure generation for the case when a noise modulation of the laser beam or/and the initial phase shift $u(x, y, 0)$ takes place.

\subsection{Student predictions about the structure behavior and test of hypothesis}

Similiarly, the TIP foresees that the student must predict the pattern evolution in time under stipulated conditions and test hypothesis. For example, the student must predict the possibility of a hysteresis of the multipetal structure configuration under the variation of the angle $\Delta$ both from the mimimum value to the maximum and from the maximum to the minimum value.

Another example of the student forecast is concerned of the spontaneous transformation of the optical patterns. This phenomenon was named by the authors the self-reorganization of the optical structures.

The student is suggested to discuss the possibility (or the impossibility) of two kinds of self - reorganization: nonstationary one and cyclic self-reorganization. The term "cyclic" means that a certain sequence of the differing optical 
patterns is repeated during some time periodically. It is obviously that similar tasks have scientific matter and form the student as optician - investigator.

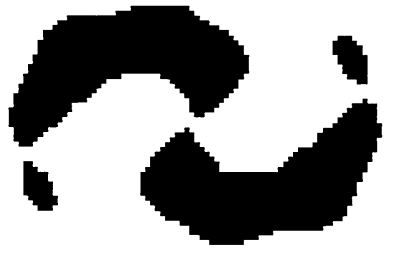

$$
0.9 \tau
$$

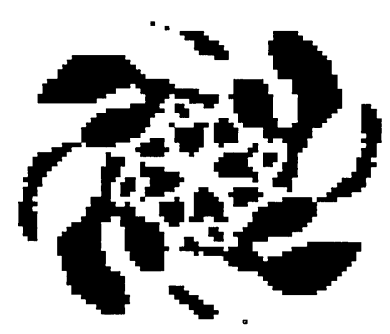

$5.8 \mathrm{\tau}$

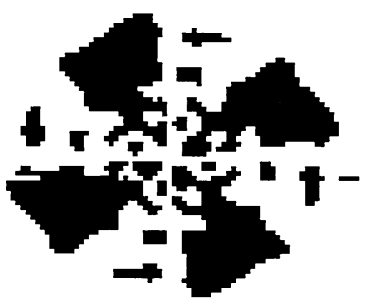

$18.5 \mathrm{t}$

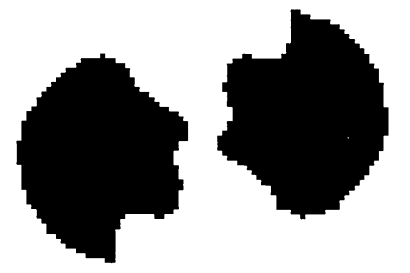

$1.8 \mathrm{\tau}$

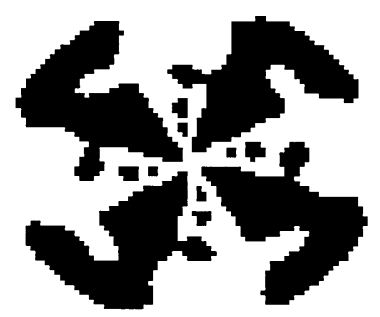

$9.3 \mathrm{~T}$

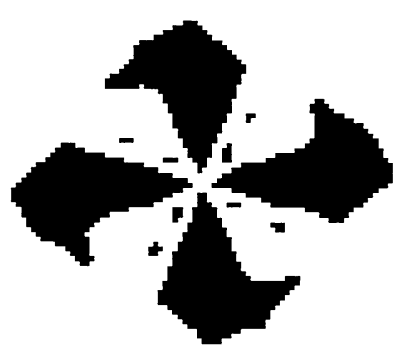

$20.4 \tau$

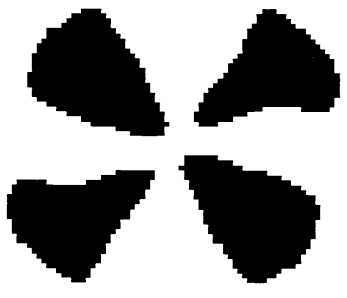

$3.0 \tau$
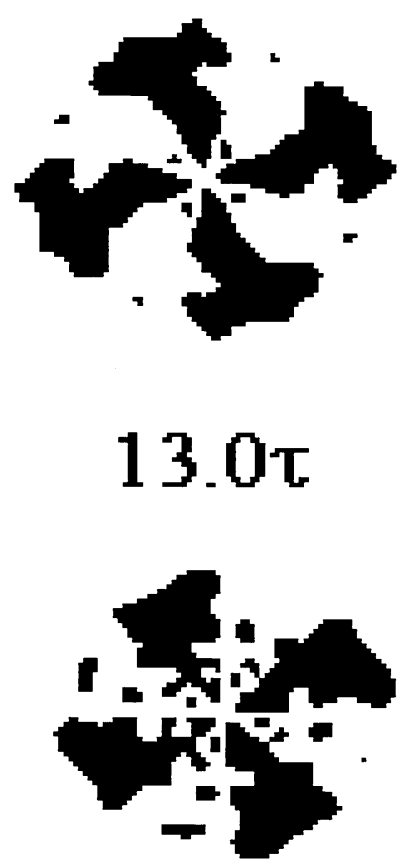

$25.6 \tau$

Fig. 8 .

Fig. 8 ilustrates the self - reorganization of the optical structures which shapes co respond to the moments $t=0.9 \tau$, $1.8 \tau$ etc. The initial conditions have form (2), $\tau=0.001 \mathrm{~s}, \Delta=50^{\circ}, \mathrm{d}=0.001, \delta=0, \alpha=0.8, \mathrm{~K}=4.08$. The patterns generated in the moments $18.5 \tau$ and $25.6 \tau$ illustrate the phenomena of the cyclic self - reorganization which period is equal approximately $7 \tau$.

The final stage of the simulation performed by the student could be named "free voyage" regime. The student can make setting of any problem, connected with the model (1) and provided the computer simulation. Of course, the student can transform the model (1) in order to exposure a new features of the optical patterns evolution and the "chaos-order" 
transition. The student must abvocate the setting of a problem, the results of the simulation and the conclusions in a form of "science propositions". Another student (who had studied the dynamics of optical structures earlier) must speak as opponent. His task is to cast some doubt on the "science propositions" trustworthiness.

\section{CONCLUSIONS}

As the authors experience shows the computer simulation of the optical synergetics processes performed by the student forms his creative thinking and develops the proffessional qualities of the optician - investigator. The student participation in the solution of the pattern selforganization problems promotes the formation of the holistic evolutional world outlook. A number of a modern thinkers and philosophers (for example, I. Prigogine and I. Stengers [5], E. Laszlo [6], E. Knyazeva and S. Kurdjumov [7,8]) beleives that holistic - evolutional consciousness is necessary for the decision of the acute problems of our changing civilization in the first place.

\section{ACNOWLEDGMENTS}

This work was performed in the frame of program "Universities of Russia" (Direction 1 "Universities as the regional centres of education, science and culture. The scientific base of state management by development of universities." Division 1.1. "The elaboration of modern university education conception") subsidizing by State Commitee on high education of Russian Federation. The authors wish to thank M.A. Vorontsov for encouragement and V.V. Sokolov for helpful critic.

\section{REFERENCES}

1. S.A. Akhamanov, M.A.Vorontsov and V.Yu. Ivanov, "Generatsiya struktur v opticheskih sistemah s dvumernoi obratnoi svyaz'yu", Novie fizicheskie printcipi opticheskoi obrabotki intormatsii, ed. by S.A. Akhmanov and M.A. Vorontsov, pp. 263-325, Nauka, Moscow, 1990.

2. M.A.Vorontsov and N.I. Zheleznih, "Poperechnaya bistabil'nost' i mul'tistabil'nost' v nelineinih opticheskih sistemah s dvumernoi obratnoi svyaz'yu", Matematicheskoe modelirovanie, Vol. 2, pp. 31-38, February 1990.

3. A.I. Arshinov, R.R. Mudarisov and B.N. Poizner, "Opticheskoe formoobrazovanie v interferometre s Kerrovskoi nelineinost'yu: vichislitel'nii eksperiment", Izvestia vuz. Fizika, pp. 102-104, June 1994.

4. A.I. Arshinov, R.R. Mudarisov, B.N. Poizner and N.A. Starova, "Optical structures caused by combined transformation of the field in the non-linear interferometer", Izvestia vuz. Fizika (Soviet Physics Journal), pp. 20-22, February 1995.

5. I. Prigogine and I. Stengers, Time, Chaos, Quantum, Progress, Moscow, 1994 (in Russian). Amsterdam, 1991

6. E. Laszlo, The Age of Bifurcation. Understanding the Changing World, Gordon and Breach Science Publishers,

7. E.N. Knyazeva and S.P. Kurdyumov, Zakoni evoljutsii i samoorganizatsii slozhnih sistem, Nauka, Moscow, 1994.

8. E.N. Knyazeva and S.P. Kurdyumov, Synergetics at the Crossroads of the Eastern and the Western Cultures, Prepr. N28 of Keldysh Institute of Appl. Math. (RAS), 1994. 